\title{
Nanoparticles: A Challenging Vehicle for Neural Stimulation
}

\author{
Elisabetta Colombo ${ }^{1 *}$, Paul Feyen ${ }^{1}$, Maria Rosa Antognazza ${ }^{2}$, Guglielmo Lanzani ${ }^{2,3}$ and \\ Fabio Benfenati ${ }^{1,4}$ \\ ${ }^{1}$ Center for Synaptic Neuroscience and Technology, Istituto Italiano di Tecnologia, Genova, Italy, ${ }^{2}$ Center for Nano Science \\ and Technology, Istituto Italiano di Tecnologia, Milan, Italy, ${ }^{3}$ Department of Physics, Politecnico di Milano, Milan, Italy, \\ ${ }^{4}$ Department of Experimental Medicine, Università di Genova, Genova, Italy
}

OPEN ACCESS

Edited by:

Michele Giugliano,

University of Antwerp, Belgium

Reviewed by:

Anja Kunze,

University of California, Los Angeles,

USA

Antonina Monaco,

University of Antwerp, Belgium

*Correspondence:

Elisabetta Colombo elisabetta.colombo@iit.it

Specialty section: This article was submitted to

Neural Technology,

a section of the journal

Frontiers in Neuroscience

Received: 30 December 2015 Accepted: 04 March 2016 Published: 23 March 2016

Citation:

Colombo E, Feyen P, Antognazza MR, Lanzani $G$ and Benfenati F (2016) Nanoparticles: A Challenging Vehicle for Neural Stimulation.

Front. Neurosci. 10:105. doi: 10.3389/fnins.2016.00105
Neurostimulation represents a powerful and well-established tool for the treatment of several diseases affecting the central nervous system. Although, effective in reducing the symptoms or the progression of brain disorders, the poor accessibility of the deepest areas of the brain currently hampers the possibility of a more specific and controlled therapeutic stimulation, depending on invasive surgical approaches and long-term stability, and biocompatibility issues. The massive research of the last decades on nanomaterials and nanoscale devices favored the development of new tools to address the limitations of the available neurostimulation approaches. This mini-review focuses on the employment of nanoparticles for the modulation of the electrophysiological activity of neuronal networks and the related transduction mechanisms underlying the nanostructure-neuron interfaces.

Keywords: nanoparticle, neural activity modulation, neurostimulation, neural prosthetics, nanobeads

\section{INTRODUCTION}

In 1939 it was reported that "Nervous messages are invariably associated with an electrical change known as the action potential," representing day-one of electrophysiology and the dawning of neural stimulation (Hodgkin and Hukley, 1939). Nearly 40 years later, cochlear implants for instance were commercially available, successfully providing functional hearing to thousands of deafened people. Along the development of conventional electrical stimulation techniques, less invasive methodologies such as transcranial magnetic or ultrasonic stimulation have been employed for motor rehabilitation or therapy of neuropsychiatric disorders (Dayan et al., 2013; Legon et al., 2014; Noda et al., 2015). A very recent advancement for the modulation of neuronal activity is the use of light stimulation (Antognazza et al., 2014). Illumination of neurons can affect their activity either by light-induced temperature raise, exploiting the intrinsic physical, and chemical dynamics of neuronal membranes (Duke et al., 2013; Farah et al., 2013; Martino et al., 2015), or by photovoltaic interfaces that transduce light into an electrical stimulation (Ghezzi et al., 2011, 2013; Mandel et al., 2013). In addition, optogenetics has shown an innovative potential to interrogate neural circuits with unprecedented precision and specificity (Packer et al., 2013) and treat several diseases such as hereditary blindness, epilepsy, or Parkinson's disease (Busskamp and Roska, 2011) with the possibility of wireless implants for chronic light stimulation (Montgomery et al., 2015).

Considering the overall historical timeline, neural stimulation has developed at the pace of silicon technology and its progressively cutting-edge miniaturization. The coming out of the socalled Michigan and later Utah microelectrode arrays demonstrated in fact that neuroscience theranostics could take advantage of implantable neural probes able to target multiple brain areas with high spatial resolution (Wise et al., 1970; Jones et al., 1992). The downscaling and development 
of stimulation electrodes and interfaces is still an actual challenge for the scientific community, recently encouraged by the success obtained in clinical cases of vision restoration (Ho A. C. et al., 2015; Stingl et al., 2015) or deep brain stimulation (DBS) treatment of neural disorders (McIntyre et al., 2015; Schoenberg et al., 2015).

In the last decade, the scientific community described nanoscale neural interfaces as a valuable tool for drug delivery or monitoring of neuronal activity (Kotov et al., 2009; Luan et al., 2014). State of the art nanoelectrodes were successfully employed to localize epileptic foci in mice (Kang et al., 2014), while plasmonic nanoantennas combined the monitoring of extracellular activity with enhanced chemical analysis of neuronal cultures (Dipalo et al., 2015). Nonetheless, nanoelectrodes technology is not yet mature enough to provide a reliable stimulation or to flawlessly couple biocompatibility and neurointerfacing issues to the edge of fabrication techniques.

Freestanding nanoparticles (NPs) face instead moderate problems concerning processing procedures, relying on established fabrication techniques (e.g., chemical, colloidal synthesis), or more inorganic-oriented techniques (epitaxial growth or vapor deposition). Notwithstanding the preservation of a nanoscale dimension together with an easily functionalized surface, NPs raise concerns about their toxicity. Although, many aspects need further investigation, it has been demonstrated that the physicochemical properties like size and shape, surface charge or composition play a crucial role. Moreover, NPs lead frequently to the generation of reactive oxygen species that cause noxious secondary effects like DNA damage, inhibition of cell growth, and mitochondrial functional loss, all eventually promoting cell death (Kang et al., 2015). Drug delivery has also been extensively pursued with NPs of a wide variety of compositions, from silica to graphene oxide (Hu et al., 2015; Xiao et al., 2015) and from liposomes to biodegradable polymers (Wei et al., 2015; Zou et al., 2015). Another extensive use of NPs deals with hyperthermal therapies for cancer treatment that mostly exploits the magnetic susceptibility of metal NPs (Hauser et al., 2015). Recently, NPs embedded into conductive polymers have been reported to act as topographic guidance for neurons (Ho D. et al., 2015), while carbon-based materials among other nanostructures (Polak and Shefi, 2015) have been shown to be an excellent tool to drive nerve tissue repair and cell differentiation (Fabbro et al., 2013).

The landscape of NPs applications in neural interfaces is vast and beyond the scope of this report, but undeniably showing the focus that lately research attributed to nanomedicine. Nanotechnology and neuroscience are still struggling to find a compromise between toxicity, functionality, and access to the brain from the periphery for the methodologies based on NPs. Overcoming the physical constraints of other stimulation techniques, such as invasive surgical implants or genetic modifications, would be a major breakthrough in therapeutic stimulation.

Given these premises, we will focus this mini-review on the main NPs-based techniques employed so far to induce changes in neuronal activity and on the origin of the phenomena responsible for neural interfacing with these nanomaterials.

\section{THERMAL STIMULATION}

Temperature variations induce a perturbation of neuronal activity originating either from the intrinsic properties of the plasma membrane or from the temperature sensitivity of membrane proteins. Spatially localized temperature gradients on neuronal tissues were reported to be responsible for the increase in membrane capacitance and the consequent trigger of action potential firing (Shapiro et al., 2012; Duke et al., 2013). On the contrary, slow and prolonged heating is known to inhibit neuronal activity due to a predominant contribution of ionic channel modulation (Duke et al., 2013). In addition, the specific temperature sensitivity of the transient receptor potential vanilloid (TRPV) (Caterina et al., 1997) constitutes another extensively studied trigger of neuronal activity.

Gold nanoparticles (AuNPs) and nanorods (AuNRs) exposed to visible or near infrared (NIR) stimuli express surface plasmon resonance that is partly converted into thermal dissipation. This phenomenon has recently been reported to mediate highly localized heat-induced changes in neuronal membrane capacitance (Paviolo and Stoddart, 2015). The thermal transduction mediated by AuNPs and AuNRs proved to be a versatile tool to affect neuronal activity, triggering membrane depolarization from 0.025 to $25 \mathrm{~ms}$ illumination pulses (Yong et al., 2014) and action potential firing up to $200 \mathrm{~Hz}$ stimulation frequencies (Carvalho-de-Souza et al., 2015), given a surface functionalization to minimize thermal damage and aggregation. Neuronal excitation has been similarly reported using AuNRs to activate temperature-sensitive channels in the rat sciatic nerve in vivo upon NIR illumination, where a temperature increase of $6^{\circ} \mathrm{C}$ resulted in 5.7 times higher neuronal responsivity (Eom et al., 2014). Photo-Absorber Induced Neural-Thermal Stimulation (PAINTS) has provided another interesting way to excite neurons, exploiting the sub-millisecond thermal transients experienced by cells around micrometric fluorescent dyes upon optical stimulation (Farah et al., 2013).

Interestingly, PEGylated AuNRs were also demonstrated to effectively drive thermal inhibition of the spiking activity of cultured neurons exposed to prolonged $785 \mathrm{~nm}$ laser pulses (Yoo et al., 2014). NRs bound to neuronal membranes contributed significantly more to the photothermal silencing than the ones suspended in the medium prior to binding, demonstrating once more the high potential for targeted stimulation. Although, the mechanisms underlying the photothermal stimulation need further investigations, an involvement of thermo-sensitive $\mathrm{K}^{+}$ channel TREK-1 is suggested to be implicated.

A similar feature was reported by using thin films of photovoltaic semiconductor polymers, which exerted both depolarizing and hyperpolarizing effects on cell lines and neurons upon illumination as a function of the illumination time (Ghezzi et al., 2013; Martino et al., 2015; Feyen et al., 2016). We think these reports provided the foundation for a downscaling of photovoltaic polymeric films to in vivo injectable NPs for neural stimulation.

Neuronal stimulation has also been achieved by inducing a localized temperature raise through the heating of NPs subjected to a magnetic field. Magnetic stimulation in the macro-scale is in 
fact a common tool in neurophysiology thanks to the possibility to reach deep areas of the brain tissue. Manganese ferrite NPs have been used to generate a behavioral response in live worms upon magnetic-induced temperature rise of $14^{\circ} \mathrm{C}$ (Huang et al., 2010). Given the prolonged duration of the stimulation and the evoked temperature increase, it is believed that the activation of TRPV1 channels by the magnetothermal stimulation represent the major mechanism involved in this effect. Indeed, in a recent work, Chen et al. (2015) showed the co-localization of transfected TRPV1 channels and neuronal activation upon magnetic field application in mice injected with iron oxide NPs by in vivo imaging of c-Fos, an immediate early gene that is upregulated by neuronal activation.

Magnetic fields compete with ultrasounds (US) as noninvasive and low toxicity sources for therapeutic tissue stimulation. A novel thermal stimulation which is also worth mentioning has in fact exploited nano-piezoelectric transducers to modulate $\mathrm{Ca}^{2+}$ channels activity in SH-SY5Y neuroblastoma cells upon $1 \mathrm{MHz}$ US stimulation (Marino et al., 2015).

\section{ELECTRIC AND ELECTROMAGNETIC STIMULATION}

Energy transduction pathways of high interest for neurostimulation are those resulting in changes in neural tissue's local electric fields. Traditionally, such approaches have been carried out using metal electrodes in contact with cells to achieve a capacitive coupling or by transcranial magnetic stimulation, which couples high intensity magnetic fields to local electrical currents in neural systems. NPs confer to these techniques a major potential for pushing forward the attainable spatial resolution, thanks to the possibility to colocalize electromagnetic fields with the NPs, and widening the penetration depth of these techniques, thanks to a possible NPs' delivery through peripheral injection or their capability to cross the blood brain barrier.

Several approaches demonstrated to be effective in stimulating neurons via photopotential/current generation from visible or infrared light absorbed in quantum confined NPs, quantum dots (QDs), and QD films. Films of HgTe QDs in conjunction with poly(Diallyl dimethyl ammonium) chloride (PDDA) were interfaced to a model neuron cell line, which showed induced action potential firing $\left(532 \mathrm{~nm}, 500 \mathrm{~ms}\right.$ pulses @ $800 \mathrm{~mW} / \mathrm{cm}^{2}$ ) nominally attributed to a resistive coupling of the neurons with the NPs interface (Pappas et al., 2007). Another report highlighted the modifications of neuronal membrane ionic conductance by induced electric dipole generation upon photostimulation of CdSe and CdTe QD films and micro-probes (Lugo et al., 2012).

Nanostructured PbSe films on glass microtips have also been reported to effectively depolarize neurons thanks to a NIRinduced local electric field (Zhao et al., 2009). More recently, semiconducting NRs on carbon nanotube (CNT) surfaces were exploited to obtain enhanced charge separation, and effectively stimulated action potential firing in blind chick retinas upon $405 \mathrm{~nm}$ illumination (Bareket et al., 2014). The latter finding not only demonstrates the potential of the platform for vision recovery, but it also represents to date the system for neural photostimulation that employs quantum confinement with the lowest stimulation threshold $\left(3 \mathrm{~mW} / \mathrm{cm}^{2}\right)$.

The organic photovoltaic blend composed by poly(3hexylthiophene) and phenyl-C61-butyric acid methyl ester (P3HT:PCBM) deposited on a conducting glass substrate evoked the generation of action potentials in cultured neurons upon short pulses of green light illumination (Ghezzi et al., 2011). A similar interface, composed by $\mathrm{P} 3 \mathrm{HT}$ on conductive glass, was able instead to trigger the excitability of explanted dystrophic retinas in response to $10 \mathrm{~ms}$ light pulses down to $1 \mu \mathrm{W} / \mathrm{mm}^{2}$ (Ghezzi et al., 2013).

Recently, another application that exploits NP films as neural interface has been reported for optogenetic activation. While infrared light allows deep penetration into the brain, no specific infrared-sensitive optogenetics opsins are available. Thus, a promising strategy to circumvent this problem is the use of upconverting NPs that allow localized emission of visible light, matching opsin's peak absorption wavelength. Light pulses of $980 \mathrm{~nm}$ delivered to $\mathrm{NaYF}_{4}: \mathrm{Yb}^{3+} / \mathrm{Tm}^{3+}$ particles were sufficient to activate channelrhodopsin-2 (ChR2) and the subsequent action potential firing in hippocampal neurons grown onto the interface (Shah et al., 2015). Thanks to the tunable absorption spectrum of the NPs, the versatility of such system was reported by the activation and firing of neurons expressing other opsins, such as C1V1 or mVChR1 (Hososhima et al., 2015).

Notably, all optically driven effects involving charge separation to drive neural stimulation have thus far been carried out on NPs deposited in form of thin films. Although, very promising, further characterization and development of these nanostructures is needed to make their introduction to neural systems in vivo less invasive, for example by testing the possibility to inject them directly from their colloidal form while maintaining effectiveness in neural stimulation.

Magnetic nanoparticles are also emerging as neurostimulation transducers for the control of neural activity through locally generated magnetic and/or electric field. Recently, $\mathrm{CoFe}_{2} \mathrm{O}_{4}$ $\mathrm{BaTiO}_{3}$ NPs were injected into the bloodstream of mice and brought across the blood-brain barrier by exploiting the dragging force of a permanent magnet. A low energy a.c. magnetic field (100 Oe @ 0-20 Hz) was then able to modulate the brain activity as recorded by electroencephalography (Guduru et al., 2015). This novel method to wirelessly elicit neuronal activation has opened a new page in nanomedicine studies, although further investigations on the specificity and mechanisms of neural activation are needed.

\section{CHEMICAL AND MECHANICAL STIMULATION}

The use of organic and inorganic low-dimensionality systems for controlled photo- and electro-chemical release of biological agents both in vitro and in vivo has been widely reported (Gendelman et al., 2015). The main advantages offered by NPs consist in the possibility to mediate transport of 
chemotherapeutics across the blood-brain barrier, to combine multifunctional constructs comprising both diagnostic and therapeutic agents, and to ensure high chemical specificity and spatial resolution. A detailed review of the numerous approaches proposed in this field is well beyond the scope of this mini-review. However, it is important to mention that, in the large majority of reports, NPs acted as mere physical carriers of biological agents, without covering specific functional roles in the chemical interaction with cells and tissues. Nevertheless, one notable example in the direction of active chemical transducers made use of polypyrrole (ppy) NPs embedded in microscale composite hydrogels for remotely controlled release of biomolecules (Li et al., 2015). In this case, ppy-NPs acted as light-sensitive, photo-thermal transducers, which released neurotransmitters (e.g., glutamate) upon NIR excitation. While taking benefit from the nanostructured system, this approach is still hampered in chronic experiments by the impossibility to replenish the microgel within a living organism.

Finally, a huge application potential for NPs was proposed in the field of mechano-transduction, i.e., in the conversion of a mechanical stimulus into an electrochemical effect (Gillespie and Walker, 2001). The mechanism underlying this phenomenon is yet to be fully understood, but it is thought to be mediated by stretch-activated ion channels, present in all cell types and directly influenced by the presence of mechanical forces acting on the cellular membrane. Importantly, mechano-transduction is currently believed to be involved in signal transduction of neurons and astrocytes (Oliet and Bourque, 1993). Recently, a number of techniques have been reported that allow NPsinduced mechanical forces to be applied to specific receptors on the surface of cultured cells, modulating mechanosensitive ion channels activity, cytoskeletal mechanics, or growth factor release (Hughes et al., 2005; Sensenig et al., 2012; Kunze et al., 2015). Magnetic beads, in particular, were attached to integrin receptors or to specific antibodies on the surface of substrateadherent cells. A high-gradient magnetic field was able to drag the particles in a given direction, exerting a localized deformation of the plasma membrane (Dobson, 2008). With a similar approach, ferromagnetic microparticles characterized by a fixed magnetization direction were attached to the cell surface through the use of specific ligand coatings. A weak external magnetic field resulted in a slight twisting of the particles and a subsequent torque onto the cell membrane, showing that forces within a few $\mathrm{pN}$ could initiate outgrowth and elongation of neurites or signaling transduction (Fass and Odde, 2003; Steketee et al., 2011). In a more recent work, cubic magnetic nanoparticles $(\mathrm{ZnFeO})$ were shown to exert mechanical forces in the order of $\mathrm{pN}$ and to efficiently and reversibly modulate the gating of mechano-sensitive ion channels at the level of single ear hair cells in frogs, with unprecedented temporal $(100 \mu \mathrm{s})$ and spatial resolution (Lee et al., 2014). Finally, induction of $\mathrm{Ca}^{2+}$ influx in cortical neurons incubated with starch- and chitosan-coated magnetic NPs have been exerted by a nanomagnetic force stimulation (Tay et al., 2016). A $20 \%$ change in calcium influx upon stimulation and an increase in firing activity were justified by the implication of mechano-sensitive ion channels. These results highlight another promising route for remote control over neuronal activity with a wireless and non-invasive magnetic force.

\section{CONCLUDING REMARKS}

In this mini-review, we presented the state of the art neurostimulation techniques using NPs and NP-enhanced surfaces. We have described three main classes of neuroninterface interactions (see Table 1), provided that many of the proposed mechanisms need further investigations and that, in some cases, the nature of the interaction stands on multiple phenomena.

In addition to the ability of modulating neuronal activity, a key issues critical for the application of these approaches to neuro-prosthetics are the possible sources of toxicity or tissue damage. One possible way to reduce the noxious impact of NPs in neural tissues is the functionalization of the surface, a procedure that was adopted by all the reports discussed here, and demonstrated also to improve either the Z-potential, the co-localization of the NPs with the tissue, or the specificity of the functional outcome. Overheating could be a harmful general effect related to any kind of energy release in tissues and organs and may represent a potential threat for NPs neural stimulation. Another possible noxious agent is represented by the generation of chemical reactants, like oxygen species, that could interfere with the viability of cells and tissues. The incubation time and the size of the NPs play finally another important role in determining the level of damage associated with the stimulation method according to the cells internalization dynamics. Interestingly, most of the reports described here experiment indeed acute administration of NPs, leaving the question open about the efficacy upon chronic exposure.

In addition, some of the presented techniques interestingly rely on the use of optogenetics to enhance or drive the NPsinduced stimulation. The application of optogenetics to humans currently suffers of drawbacks and uncertainties regarding the safety and long-term efficacy of gene therapy. Moreover, optogenetics alone is a strong competitor for NPs driven neurostimulation in terms of specificity and spatial resolution.

It is also worth mentioning the lack for a suitable way to electrically stimulate by means of NPs, if they are not in contact with an anodic/cathodic circuit, although the possibility may exist to modulate neuronal activity by the generation of local electric fields by un-contacted NPs (Zhao et al., 2009).

Another important feature emerging from this mini-review is that most of the effective ways to trigger time-controlled and targeted changes in the electrophysiological state of neuronal membranes and their consequent action potential firing has been achieved with inorganic nanoparticles.

In conclusion, NPs represent a novel transduction platform that matches the increasing demand for spatial and targeted specificity in the treatment of neurological disorders. However, very few in vivo trials have been produced so far exploiting NPs as vehicle for the modulation of neural activity, demonstrating that the research in this field is still at a very early stage. Nevertheless, 


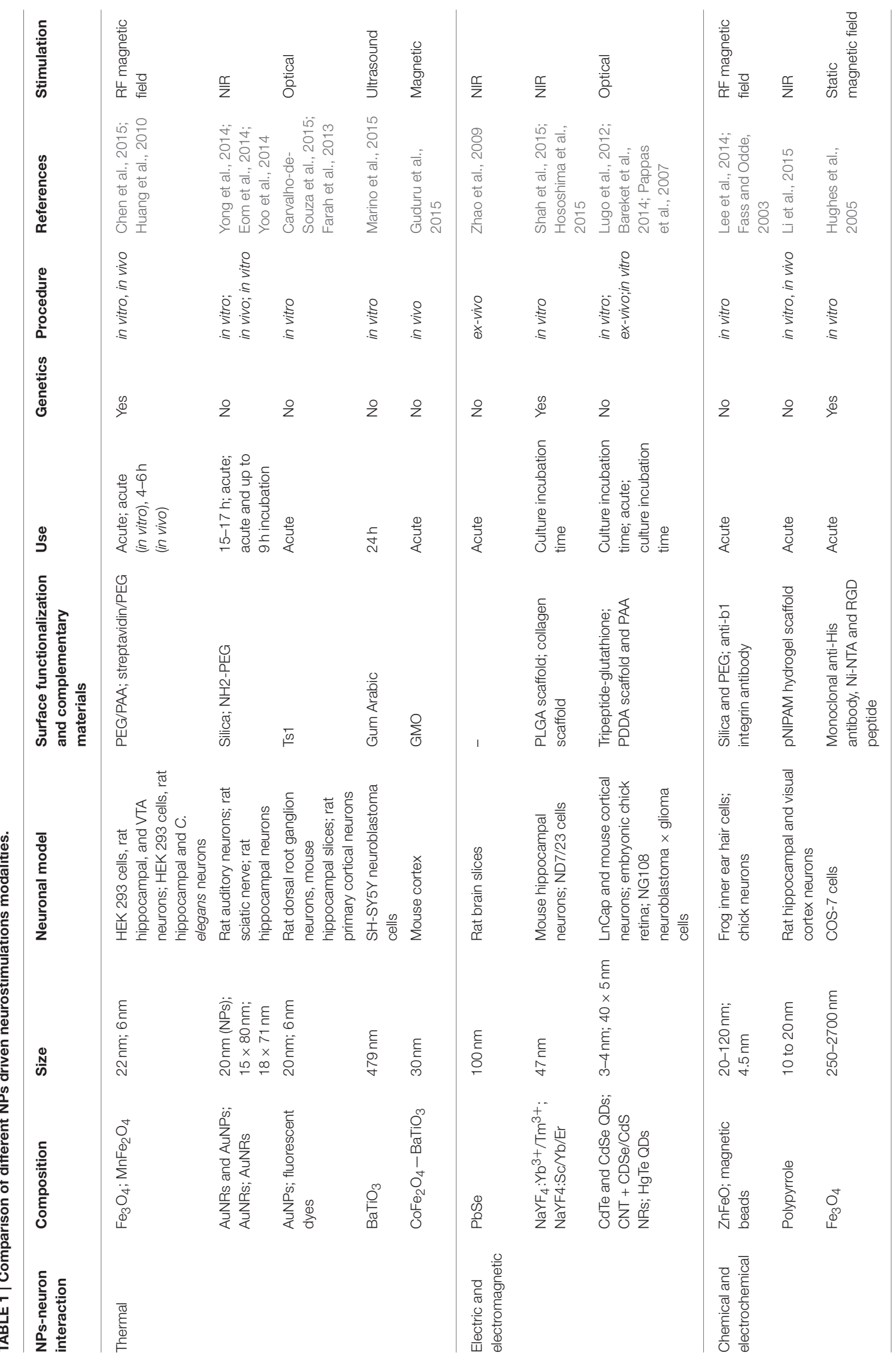


we believe that the striking results in neural stimulation by injection of magnetic NPs or AuNRs show the potential impact of these technologies as new therapeutic tools for neuronal injury, neuropathologies or chronic pain, just to mention a few. Next steps toward medical practice will include a more robust understanding of the mechanisms underlying the stimulation procedures and reliable chronic exposure tests.

\section{REFERENCES}

Antognazza, M. R., Martino, N., Ghezzi, D., Feyen, P., Colombo, E., Endeman, D., et al. (2014). Shedding light on living cells. Adv. Mater, 27, 7662-7669. doi: 10.1002/adma.201403513

Bareket, L., Waiskopf, N., Rand, D., Lubin, G., David-Pur, M., Ben-Dov, J., et al. (2014). Semiconductor nanorod-carbon nanotube biomimetic films for wire-free photostimulation of blind retinas. Nano Lett. 14, 6685-6692. doi: $10.1021 / \mathrm{nl} 5034304$

Busskamp, V., and Roska, B. (2011). Optogenetic approaches to restoring visual function in retinitis pigmentosa. Curr. Opin. Neurobiol. 21, 942-946. doi: 10.1016/j.conb.2011.06.001

Carvalho-de-Souza, J. L., Treger, J. S., Dang, B., Kent, S. B. H., Pepperberg, D. R., and Bezanilla, F. (2015). Photosensitivity of neurons enabled by cell-targeted gold nanoparticles. Neuron 86, 207-217. doi: 10.1016/j.neuron.2015.02.033

Caterina, M., Schumacher, M., Tominaga, M., Rosen, T., Levine, J., and Julius, D. (1997). The capsaicin receptor: a heat-activated ion channel in the pain pathway. Nature 389, 816-824. doi: 10.1038/39807

Chen, R., Romero, G., Christiansen, M. G., Mohr, A., and Anikeeva, P. (2015). Wireless magnetothermal deep brain stimulation. Science 347, 1477-1480. doi: 10.1126/science. 1261821

Dayan, E., Censor, N., Buch, E. R., Sandrini, M., and Cohen, L. G. (2013). Noninvasive brain stimulation: from physiology to network dynamics and back. Nat. Neurosci. 16, 838-844. doi: 10.1038/nn.3422

Dipalo, M., Messina, G. C., Amin, H., La Rocca, R., Shalabaeva, V., Simi, A., et al. (2015). 3D plasmonic nanoantennas integrated with MEA biosensors. Nanoscale 7, 3703-3711. doi: 10.1039/C4NR05578K

Dobson, J. (2008). Remote control of cellular behaviour with magnetic nanoparticles. Nat. Nanotechnol. 3, 139-143. doi: 10.1038/nnano.2008.39

Duke, A. R., Jenkins, M. W., Lu, H., McManus, J. M., Chiel, H. J., and Jansen, E. D. (2013). Transient and selective suppression of neural activity with infrared light. Sci. Rep. 3:2600. doi: 10.1038/srep02600

Eom, K., Kim, J., Choi, J. M., Kang, T., Chang, J. W., Byun, K. M., et al. (2014). Enhanced infrared neural stimulation using localized surface plasmon resonance of gold nanorods. Small 10, 3853-3857. doi: 10.1002/smll.201400599

Fabbro, A., Prato, M., and Ballerini, L. (2013). Carbon nanotubes in neuroregeneration and repair. Adv. Drug Deliv. Rev. 65, 2034-2044. doi: 10.1016/j.addr.2013.07.002

Farah, N., Zoubi, A., Matar, S., Golan, L., Marom, A., Butson, C. R., et al. (2013). Holographically patterned activation using photo-absorber induced neural-thermal stimulation. J. Neural Eng. 10:056004. doi: 10.1088/17412560/10/5/056004

Fass, J. N., and Odde, D. J. (2003). Tensile force-dependent neurite elicitation via anti-betal integrin antibody-coated magnetic beads. Biophys. J. 85, 623-636. doi: 10.1016/S0006-3495(03)74506-8

Feyen, P., Colombo, E., Endeman, D., Nova, M., Laudato, L., Martino, N., et al. (2016). Light-evoked hyperpolarization and silencing of neurons by conjugated polymers. Sci. Rep. 6:22718. doi: 10.1038/srep22718

Gendelman, H. E., Anantharam, V., Bronich, T., Ghaisas, S., Jin, H., Kanthasamy, A. G., et al. (2015). Nanoneuromedicines for degenerative, inflammatory, and infectious nervous system diseases. Nanomed. Nanotechnol. Biol. Med. 11, 751-767. doi: 10.1016/j.nano.2014.12.014

Ghezzi, D., Antognazza, M. R., Dal Maschio, M., Lanzarini, E., Benfenati, F., and Lanzani, G. (2011). A hybrid bioorganic interface for neuronal photoactivation. Nat. Commun. 2, 166. doi: 10.1038/ncomms1164

\section{AUTHOR CONTRIBUTIONS}

EC, MA, and PF contributed to the conception of the work and the writing of the manuscript. FB and GL revised the work critically for important intellectual content and helped correcting the final version of the manuscript. All authors read and approved the final version of the manuscript.

Ghezzi, D., Antognazza, M. R., Maccarone, R., Bellani, S., Lanzarini, E., Martino, N., et al. (2013). A polymer optoelectronic interface restores light sensitivity in blind rat retinas. Nat. Photonics 7, 400-406. doi: 10.1038/nphoton.2 013.34

Gillespie, P. G., and Walker, R. G. (2001). Molecular basis of mechanosensory transduction. Nature 413, 194-202. doi: 10.1038/35093011

Guduru, R., Liang, P., Hong, J., Rodzinski, A., Hadjikhani, A., Horstmyer, J., et al. (2015). Magnetoelectric spin on stimulating the brain. Nanomedicine 10, 2051-2061. doi: 10.2217/nnm.15.52

Hauser, A. K., Wydra, R. J., Stocke, N. A., Anderson, K. W., and Hilt, J. Z. (2015). Magnetic nanoparticles and nanocomposites for remote controlled therapies. J. Control. Release 219, 76-94. doi: 10.1016/j.jconrel.2015.09.039

Ho, A. C., Humayun, M. S., Dorn, J. D., da Cruz, L., Dagnelie, G., Handa, J., et al. (2015). Long-term results from an epiretinal prosthesis to restore sight to the blind. Ophthalmology 122, 1547-1554. doi: 10.1016/j.ophtha.2015.04.032

Ho, D., Zou, J., Chen, X., Munshi, A., Smith, N. M., Agarwal, V., et al. (2015). Hierarchical patterning of multifunctional conducting polymer nanoparticles as a bionic platform for topographic contact guidance. ACS Nano 9, 1767-1774. doi: $10.1021 / \mathrm{nn} 506607 \mathrm{x}$

Hodgkin, A. L., and Hukley, A. F. (1939). Action potentials recorded from inside a nerve fibre. Nature 144, 710-711. doi: 10.1038/144710a0

Hososhima, S., Yuasa, H., Ishizuka, T., Hoque, M. R., Yamashita, T., Yamanaka, A., et al. (2015). Near-infrared (NIR) up-conversion optogenetics. Sci. Rep. 5:16533. doi: 10.1038/srep16533

Hu, Y., Li, F., Han, D., and Niu, L. (2015). Biocompatible Graphene for Bioanalytical Applications. Berlin; Heidelberg: Springer.

Huang, H., Delikanli, S., Zeng, H., Ferkey, D. M., and Pralle, A. (2010). Remote control of ion channels and neurons through magnetic-field heating of nanoparticles. Nat. Nanotechnol. 5, 602-606. doi: 10.1038/nnano. 2010.125

Hughes, S., El Haj, A. J., and Dobson, J. (2005). Magnetic micro- and nanoparticle mediated activation of mechanosensitive ion channels. Med. Eng. Phys. 27, 754-762. doi: 10.1016/j.medengphy.2005.04.006

Jones, K. E., Campbell, P. K., and Normann, R. A. (1992). A glass/silicon composite intracortical electrode array. Ann. Biomed. Eng. 20, 423-37. doi: 10.1007/BF02368134

Kang, H., Mintri, S., Menon, A. V., Lee, H. Y., Choi, H. S., and Kim, J. (2015). Pharmacokinetics, pharmacodynamics and toxicology of theranostic nanoparticles. Nanoscale 7, 18848-18862. doi: 10.1039/C5NR05264E

Kang, M., Jung, S., Zhang, H., Kang, T., Kang, H., Yoo, Y., et al. (2014). Subcellular neural probes from single-crystal gold nanowires. ACS Nano 8, 8182-8189. doi: $10.1021 / \mathrm{nn} 5024522$

Kotov, N. A., Winter, J. O., Clements, I. P., Jan, E., Timko, B. P., Campidelli, S., et al. (2009). Nanomaterials for neural interfaces. Adv. Mater. Weinheim. 21, 3970-4004. doi: 10.1002/adma.200801984

Kunze, A., Tseng, P., Godzich, C., Murray, C., Caputo, A., Schweizer, F. E., et al. (2015). Engineering cortical neuron polarity with nanomagnets on a chip. ACS Nano 9, 3664-3676. doi: 10.1021/nn505330w

Lee, J. H., Kim, J. W., Levy, M., Kao, A., Noh, S. H., Bozovic, D., et al. (2014). Magnetic nanoparticles for ultrafast mechanical control of inner ear hair cells. ACS Nano 8, 6590-6598. doi: 10.1021/nn5020616

Legon, W., Sato, T. F., Opitz, A., Mueller, J., Barbour, A., Williams, A., et al. (2014). Transcranial focused ultrasound modulates the activity of primary somatosensory cortex in humans. Nat. Neurosci. 17, 322-329. doi: $10.1038 / \mathrm{nn} .3620$ 
Li, W., Luo, R., Lin, X., Jadhav, A. D., Zhang, Z., Yan, L., et al. (2015). Remote modulation of neural activities via near-infrared triggered release of biomolecules. Biomaterials 65, 76-85. doi: 10.1016/j.biomaterials.2015.06.041

Luan, S., Williams, I., Nikolic, K., and Constandinou, T. G. (2014). Neuromodulation: present and emerging methods. Front. Neuroeng. 7:27. doi: 10.3389/fneng.2014.00027

Lugo, K., Miao, X., Rieke, F., and Lin, L. Y. (2012). Remote switching of cellular activity and cell signaling using light in conjunction with quantum dots. Biomed. Opt. Express 3, 447-454. doi: 10.1364/BOE.3.000447

Mandel, Y., Goetz, G., Lavinsky, D., Huie, P., Mathieson, K., Wang, L., et al. (2013). Cortical responses elicited by photovoltaic subretinal prostheses exhibit similarities to visually evoked potentials. Nat. Commun. 4, 1980. doi: $10.1038 /$ ncomms 2980

Marino, A., Arai, S., Hou, Y., Sinibaldi, E., Pellegrino, M., and Chang, Y. (2015). Piezoelectric nanoparticle-assisted wireless neuronal stimulation. ACS Nano 9, 7678-7689. doi: 10.1021/acsnano.5b03162

Martino, N., Feyen, P., Porro, M., Bossio, C., Zucchetti, E., Ghezzi, D., et al. (2015). Photothermal cellular stimulation in functional bio-polymer interfaces. Sci. Rep. 5:8911. doi: 10.1038/srep08911

McIntyre, C. C., Chaturvedi, A., Shamir, R. R., and Lempka, S. F. (2015). Engineering the next generation of clinical deep brain stimulation technology. Brain Stimul. 8, 21-26. doi: 10.1016/j.brs.2014.07.039

Montgomery, K. L., Yeh, A. J., Ho, J. S., Tsao, V., Mohan Iyer, S., Grosenick, L., et al. (2015). Wirelessly powered, fully internal optogenetics for brain, spinal and peripheral circuits in mice. Nat. Methods 12, 969-974. doi: 10.1038/nmeth.3536

Noda, Y., Silverstein, W. K., Barr, M. S., Vila-Rodriguez, F., Downar, J., Rajji, T. K., et al. (2015). Neurobiological mechanisms of repetitive transcranial magnetic stimulation of the dorsolateral prefrontal cortex in depression: a systematic review. Psychol. Med. 45, 3411-3432. doi: 10.1017/S0033291715001609

Oliet, S. H., and Bourque, C. W. (1993). Mechanosensitive channels transduce osmosensitivity in supraoptic neurons. Nature 364, 341-343. doi: $10.1038 / 364341 \mathrm{a} 0$

Packer, A. M., Roska, B., and Häusser, M. (2013). Targeting neurons and photons for optogenetics. Nat. Neurosci. 16, 805-815. doi: 10.1038/nn.3427

Pappas, T. C., Wickramanyake, W. M. S., Jan, E., Motamedi, M., Brodwick, M., and Kotov, N. A. (2007). Nanoscale engineering of a cellular interface with semiconductor nanoparticle films for photoelectric stimulation of neurons. Nano Lett. 7, 513-519. doi: 10.1021/nl062513v

Paviolo, C., and Stoddart, P. R. (2015). Metallic nanoparticles for peripheral nerve regeneration: is it a feasible approach? Neural Regen. Res. 10, 1065-1066. doi: 10.4103/1673-5374.160083

Polak, P., and Shefi, O. (2015). Nanometric agents in the service of neuroscience: manipulation of neuronal growth and activity using nanoparticles. Nanomedicine 11, 1467-1479. doi: 10.1016/j.nano.2015.03.005

Schoenberg, M. R., Maddux, B. N., Riley, D. E., Whitney, C. M., Ogrocki, P. K., Gould, D., et al. (2015). Five-months-postoperative neuropsychological outcome from a pilot prospective randomized clinical trial of thalamic deep brain stimulation for Tourette syndrome. Neuromodulation 18, 97-104. doi: 10.1111/ner.12233

Sensenig, R., Sapir, Y., MacDonald, C., Cohen, S., and Polyak, B. (2012). Magnetic nanoparticle-based approaches to locally target therapy and enhance tissue regeneration in vivo. Nanomedicine 7, 1425-1442. doi: 10.2217/nnm.12.109

Shah, S., Liu, J.-J., Pasquale, N., Lai, J., McGowan, H., Pang, Z. P., et al. (2015). Hybrid upconversion nanomaterials for optogenetic neuronal control. 7, 16571-16577. Nanoscale. doi: 10.1039/C5NR03411F
Shapiro, M. G., Homma, K., Villarreal, S., Richter, C.-P., and Bezanilla, F. (2012). Infrared light excites cells by changing their electrical capacitance. Nat. Commun. 3, 736. doi: 10.1038/ncomms1742

Steketee, M. B., Moysidis, S. N., Jin, X.-L., Weinstein, J. E., Pita-Thomas, W., Raju, H. B., et al. (2011). Nanoparticle-mediated signaling endosome localization regulates growth cone motility and neurite growth. Proc. Natl. Acad. Sci. U.S.A. 108, 19042-19047. doi: 10.1073/pnas.1019624108

Stingl, K., Bartz-Schmidt, K. U., Besch, D., Chee, C. K., Cottriall, C. L., Gekeler, F., et al. (2015). Subretinal Visual Implant Alpha IMS-Clinical trial interim report. Vision Res. 111, 149-160. doi: 10.1016/j.visres.2015. 03.001

Tay, A., Kunze, A., Murray, C., and Di Carlo, D. (2016). Induction of calcium influx in cortical neural networks by nanomagnetic forces. ACS Nano 10, 2331-2341. doi: $10.1021 /$ acsnano.5b07118

Wei, X., Gao, J., Zhan, C., Xie, C., Chai, Z., Ran, D., et al. (2015). Liposome-based glioma targeted drug delivery enabled by stable peptide ligands. J. Control. Release 218, 13-21. doi: 10.1016/j.jconrel.2015.09.059

Wise, K. D., Angell, J. B., and Starr, A. (1970). An integrated-circuit approach to extracellular microelectrodes. IEEE Trans. Biomed. Eng. 17, 238-247. doi: 10.1109/TBME.1970.4502738

Xiao, D., Jia, H.-Z., Ma, N., Zhuo, R.-X., and Zhang, X.-Z. (2015). A redoxresponsive mesoporous silica nanoparticle capped with amphiphilic peptides by self-assembly for cancer targeting drug delivery. Nanoscale 7, 10071-10077. doi: 10.1039/C5NR02247A

Yong, J., Needham, K., Brown, W. G. A., Nayagam, B. A., Mcarthur, S. L., Yu, A., et al. (2014). Gold-nanorod-assisted near-infrared stimulation of primary auditory neurons. Adv. Healthc. Mater. 3, 1862-1868. doi: 10.1002/adhm.201400027

Yoo, S., Hong, S., Choi, Y., Park, J., and Nam, Y. (2014). Photothermal inhibition of neural activity with near-infrared-sensitive. ACS Nano. 8, 8040-8049. doi: $10.1021 /$ nn5020775

Zhao, Y., Larimer, P., Pressler, R. T., Strowbridge, B. W., and Burda, C. (2009). Wireless activation of neurons in brain slices using nanostructured semiconductor photoelectrodes. Angew. Chemie Int. Ed. 48, 2407-2410. doi: 10.1002/anie.200806093

Zou, T., Dembele, F., Beugnet, A., Sengmanivong, L., Trepout, S., Marco, S., et al. (2015). Nanobody-functionalized PEG-b-PCL polymersomes and their targeting study. J. Biotechnol. 214, 147-155. doi: 10.1016/j.jbiotec.2015. 09.034

Conflict of Interest Statement: The authors declare that the research was conducted in the absence of any commercial or financial relationships that could be construed as a potential conflict of interest.

The reviewer AM declared that she is a postdoctoral fellow in the handling Editor's laboratory and the handling Editor states that the process nevertheless met the standards of a fair and objective review.

Copyright (c) 2016 Colombo, Feyen, Antognazza, Lanzani and Benfenati. This is an open-access article distributed under the terms of the Creative Commons Attribution License (CC BY). The use, distribution or reproduction in other forums is permitted, provided the original author(s) or licensor are credited and that the original publication in this journal is cited, in accordance with accepted academic practice. No use, distribution or reproduction is permitted which does not comply with these terms. 